\title{
A Survey of Recent Advances in Efficient Parsing for Graph Grammars
}

\author{
Invited Talk
}

\author{
Frank Drewes \\ Umeå University, Sweden
}

\begin{abstract}
Context-free graph grammars, in particular hyperedge replacement graph grammars, look back on over 30 years of history. They share many of the good properties of contextfree string languages. Unfortunately, the complexity of parsing is the big exception: early results in the field showed that even for fixed grammars, the membership problem can be NP-complete. Moreover, the known results about polynomial parsing that were obtained afterwards, while constituting nice theoretical work, seemed to be of limited practical value. This is because they were either based on very "impractical" restrictions, or the degree of the polynomial running time depended on the grammar and could thus become large.

In the current decade, the question received renewed interest because hyperedge replacement is one of the candidate formalisms for specifying semantic graphs in natural language processing. Using graph grammars in this area requires parsing algorithms that are not only polynomial in theory, but efficient in practice. Preferably, the degree of the polynomial bounding their running time should be a (small) constant independent of the grammar, or else it should depend on parameters not likely to be large. The talk will present an overview of results towards this goal, discussing their requirements, advantages, and disadvantages as well as a few possible directions for future work.
\end{abstract}

Speaker's homepage: https://www.umu.se/en/staff/frank-drewes/ 